\title{
Mortality among hip fracture patients infected with COVID-19 perioperatively
}

\author{
Johannes Fessler ${ }^{1} \cdot$ Thomas Jacobsen $^{1} \cdot$ Jes Bruun Lauritzen ${ }^{1,3}$ (D) Henrik Løvendahl Jørgensen ${ }^{2,3}$ (D)
}

Received: 2 December 2020 / Accepted: 3 March 2021 / Published online: 11 March 2021

c) Springer-Verlag GmbH Germany, part of Springer Nature 2021

\begin{abstract}
Background and purpose The outbreak of the COVID-19 pandemic has resulted in an overall decline in fractures. However, the amount of hip fractures has remained relatively stable throughout the period. The objective of this study is to investigate the impact of perioperative COVID-19 infections on mortality among hip fracture patients.

Methods A meta-analysis was performed by collecting current data available through a systematic literature search in the PubMed database. The search was performed Oct 182020.

Results The meta-analysis was conducted on a trial population consisting of 1.272 hip fracture patients with a pooled prevalence of COVID-19 of 18\%. Mortality among hip fracture patients without a perioperative COVID-19 infection was $7.49 \%$. Mortality among hip fracture patients infected with COVID-19 perioperatively was associated with an odds ratio of 6.70 [(95\% CI 4.64-9.68), $p<0.00001, I^{2}=41 \%$ ]. A sensitivity analysis showed no major impact of assumptions regarding varying definitions of COVID-19 statuses among the included studies.

Conclusion Perioperative infections with COVID-19 in hip fracture patients are correlated with a significantly increased mortality. The meta-analysis showed a pooled odds ratio of 6.70 [(95\% CI 4.64-9.68), $p<0.00001, I^{2}=41 \%$ ].
\end{abstract}

\section{Introduction}

Mortality among patients infected with COVID-19 has been shown to be correlated with older age and comorbidities such as hypertension, obesity and diabetes [1]. During the outbreak of the COVID-19 pandemic, a study from Italy, based on data from 15 national Orthopaedic and Trauma centres, found a significant decrease in both emergency surgery and elective surgery during the period from the 23rd of Feb to the 4th of Apr. However, femoral neck fractures were the only exception, as a much lesser reduction of only $15 \%$ was observed at the end of the period [2]. Hip fractures are most frequent among the elderly [3] and are correlated

Johannes Fessler and Thomas Jacobsen: Co-first authors.

Henrik Løvendahl Jørgensen

hlj@dadlnet.dk

1 Department of Orthopedic Surgery, Bispebjerg Hospital, Bispebjerg Bakke 23, 2400 KBH NV Copenhagen, Denmark

2 Department of Clinical Biochemistry, Hvidovre Hospital, Kettegård Alle 30, 2650 Hvidovre, Denmark

3 Department of Clinical Medicine, University of Copenhagen, Blegdamsvej 3B, 2200 KBH N Copenhagen, Denmark with a high level of comorbidity and mortality [4]. As hip fractures are still treated surgically during the COVID-19 pandemic, it is relevant to investigate how the virus affects the survival of this group of patients.

Several studies, with a variable number of patients, have suggested that hip fracture patients are at a higher risk of a fatal outcome during the COVID-19 pandemic [5-12]. The objective of this article is to investigate and discuss the mortality among hip fracture patients who are infected with COVID-19 perioperatively. This will be done through a meta-analysis.

\section{Materials and methods}

The PRISMA guidelines for meta-analyses have been followed [13].

\section{Search strategy}

Articles concerning mortality among hip fracture patients during the COVID-19 pandemic were selected, through a systematic literature search in the PubMed database. Thus, only articles concerning survival outcomes of patients 
exposed to hip fracture with a recorded COVID-19 status were selected. The following search string was used:

(("COVID-19" (Supplementary Concept) OR ("COVID19" OR "COVID19" OR "SARS-CoV-2" OR "severe acute respiratory syndrome virus" OR "coronavirus")).

AND

(("Hip Fractures"[Mesh]) OR ((“femoral neck" AND fractur*) OR (*trochant* AND fractur*) OR ("collum femoris" AND fractur*) OR (intertrochant* AND fractur*) OR (proximal femoral fractur*))).

The search was performed Oct 182020 and initially gave 32 results. The abstracts of these articles were screened, and relevant articles were selected for full text review. Selection was made according to the criteria listed below, which resulted in eight articles for incorporation into the metaanalysis (Fig. 1).
The "similar articles" section in Pubmed was screened but gave no additional results for incorporation into the meta-analysis.

\section{Selection criteria}

The criteria for inclusion were: (1) English or Danish text, (2) data on infection status and survival outcome, (3) trial population larger than 20 , (4) study including both confirmed infected and non-infected patients, (5) mortality measured over 14-35 days postoperatively, (6) trial population of mixed gender, (7) average age of trial population above 65 years.

Duplicate publications and articles using data from the same trial populations were excluded.
Fig. 1 Flowchart illustrating the selection process

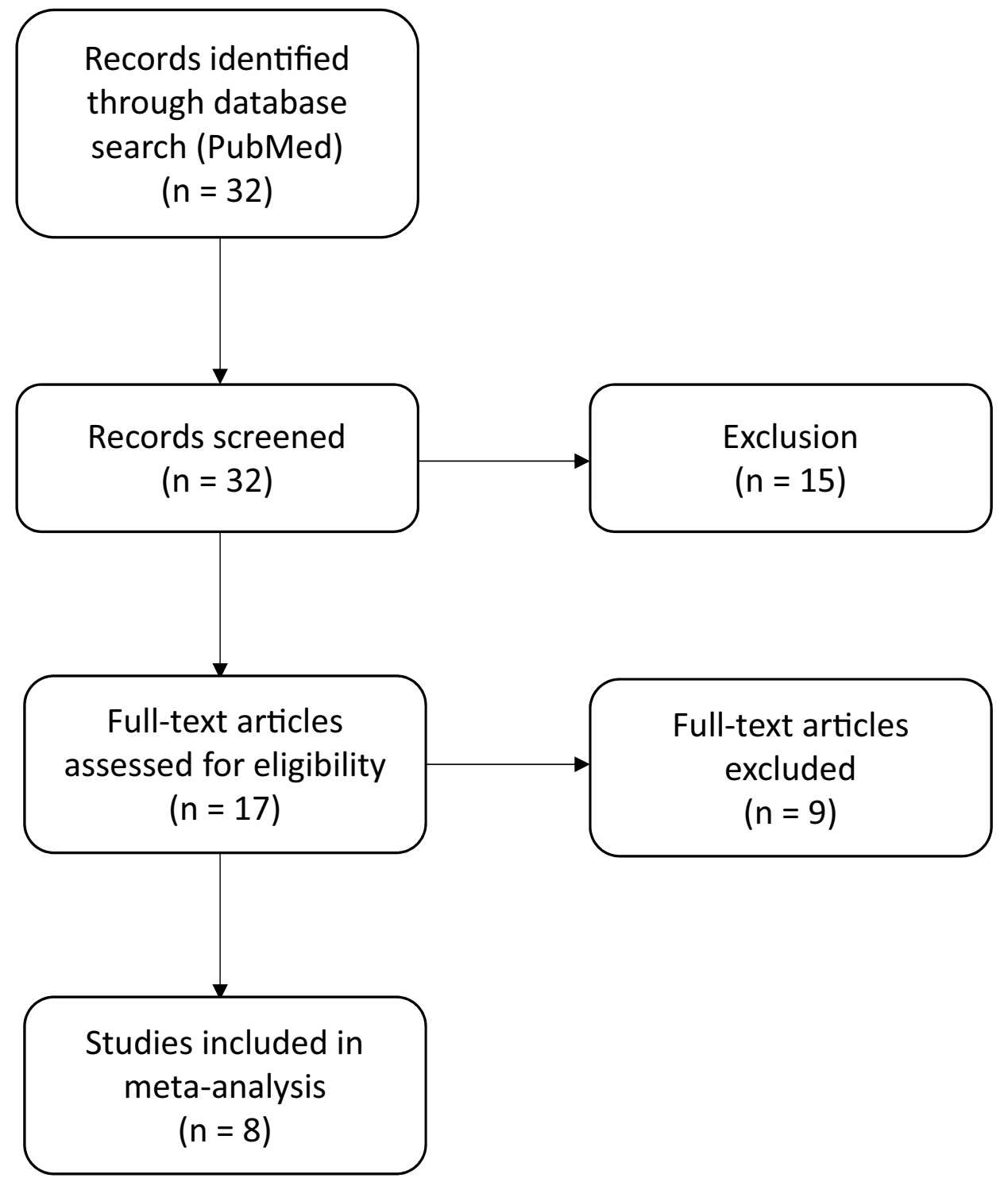




\section{Analysis}

The selection process resulted in a trial population consisting of 1272 patients with a pooled prevalence of COVID-19 of $18 \%$.

To perform the meta-analysis Review Manager 5.4 was used. Odds ratios (OR) for mortality in COVID-19 infected hip fracture patients were calculated, along with their $95 \%$ confidence intervals. $p<0.05$ was set to be significant. Review Manager automatically adjusts for zero cell counts as in the case of the Nuñez JH study, by performing the Haldane correction [14].

The test statistics $I^{2}$ was used to measure the heterogeneity across the studies. Due to a relatively low variation between the studies $\left(I^{2}=41 \%\right)$, a fixed model was chosen.

A sensitivity analysis was performed to investigate the impact of the variable definitions of COVID-19 statuses among the articles: several of the analyzed studies include patients who only showed typical symptoms of COVID19 infection (COVID-19-suspected patient) in the group of confirmed positive patients. In other studies, patients who did not show any signs of infection were not tested for COVID-19, but still added to the group of negative patients.

\section{Ethics}

According to Danish law, neither Ethical Review Board approval nor informed consent is required for this type of meta-analysis.

\section{Results}

\section{Included studies}

Table 1 shows basic characteristics of the studies, including first author, country, publication year, number of patients included, average age, number of days mortality was measured postoperatively, odds ratios and $p$ values. Figures $2-4$ illustrate the odds ratios for mortality for COVID-19 positive and negative patients (COVID +/non-COVID), for each study and the pooled odds ratios. Figures 3-4 furthermore illustrate a sensitivity analysis to test the robustness of the meta-analysis.

Table 1 Basic characteristics of the studies (all published in 2020)

\begin{tabular}{lllll}
\hline First author & Country & $\begin{array}{l}\text { Patients } \\
\text { included }\end{array}$ & $\begin{array}{l}\text { Average age } \\
\text { (years) }\end{array}$ & $\begin{array}{l}\text { Mortality } \\
\text { measured } \\
\text { postopera- } \\
\text { tively (days) }\end{array}$ \\
\hline Egol KA & USA & 138 & 83 & 30 \\
Hall AJ & UK & 317 & 80.8 & 30 \\
Kayani B & UK & 422 & 72.7 & 30 \\
LeBrun DG & USA & 59 & 85 & 35 \\
Maniscalco P & Italy & 121 & 81.1 & 21 \\
Munoz Vives & Spain & 136 & 85 & 14 \\
JM & Spain & 36 & 88.4 & $\begin{array}{c}\text { Until dis- } \\
\text { charged }\end{array}$ \\
Nunez JH & UK & 43 & 81.6 & 30 \\
Thakrar A & & & & \\
\hline
\end{tabular}

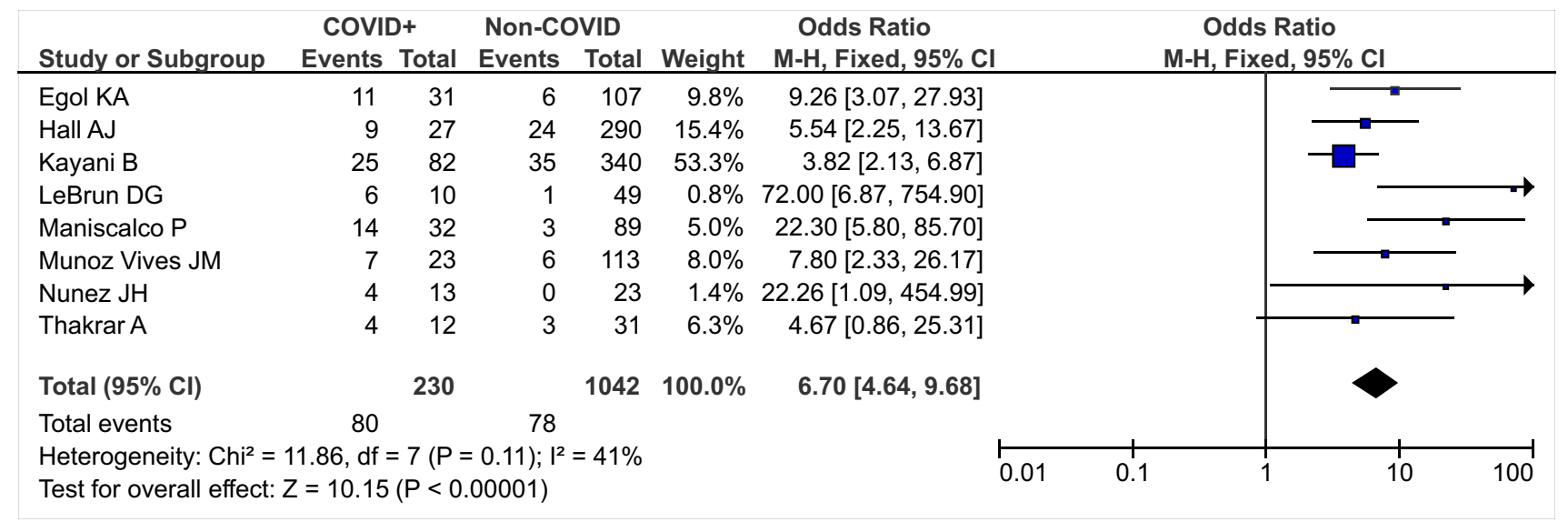

Fig. 2 Odds ratios for mortality in patients with COVID-19 versus non-COVID illustrated in a Forest plot. The pooled estimate was generated using a fixed effect model 


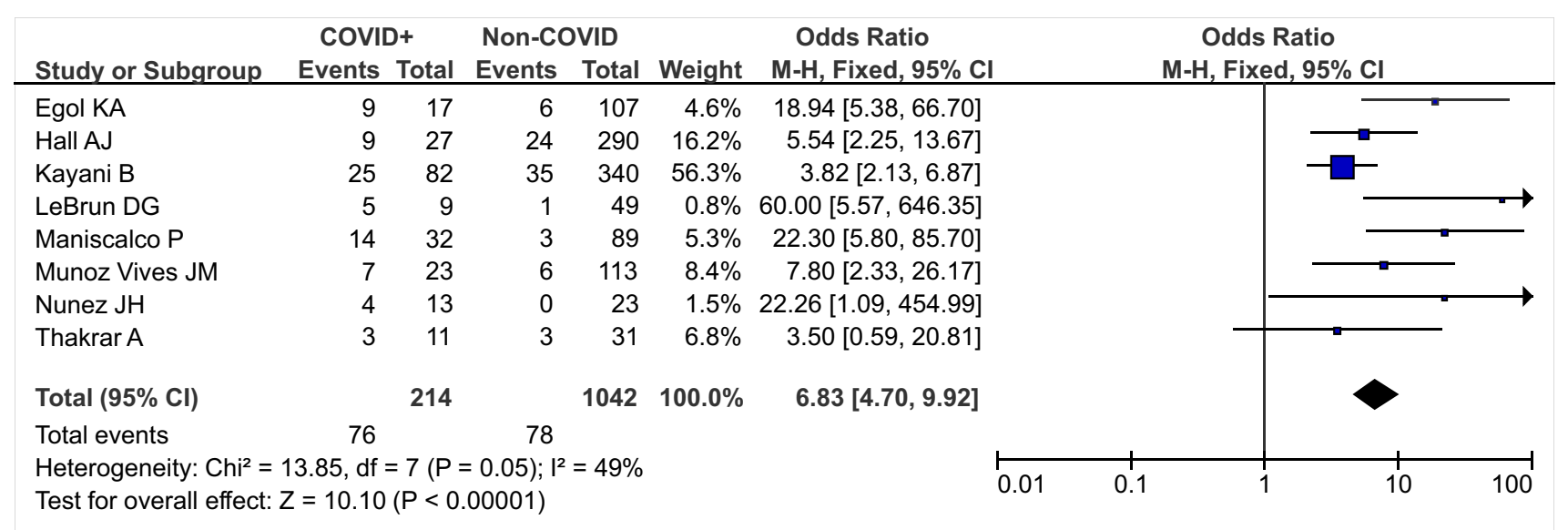

Fig. 3 Sensitivity analysis-Odds ratios for mortality in patients with COVID-19 (without COVID-19 suspected) versus non-COVID illustrated in a Forest plot. The pooled estimate was generated using a fixed effect model

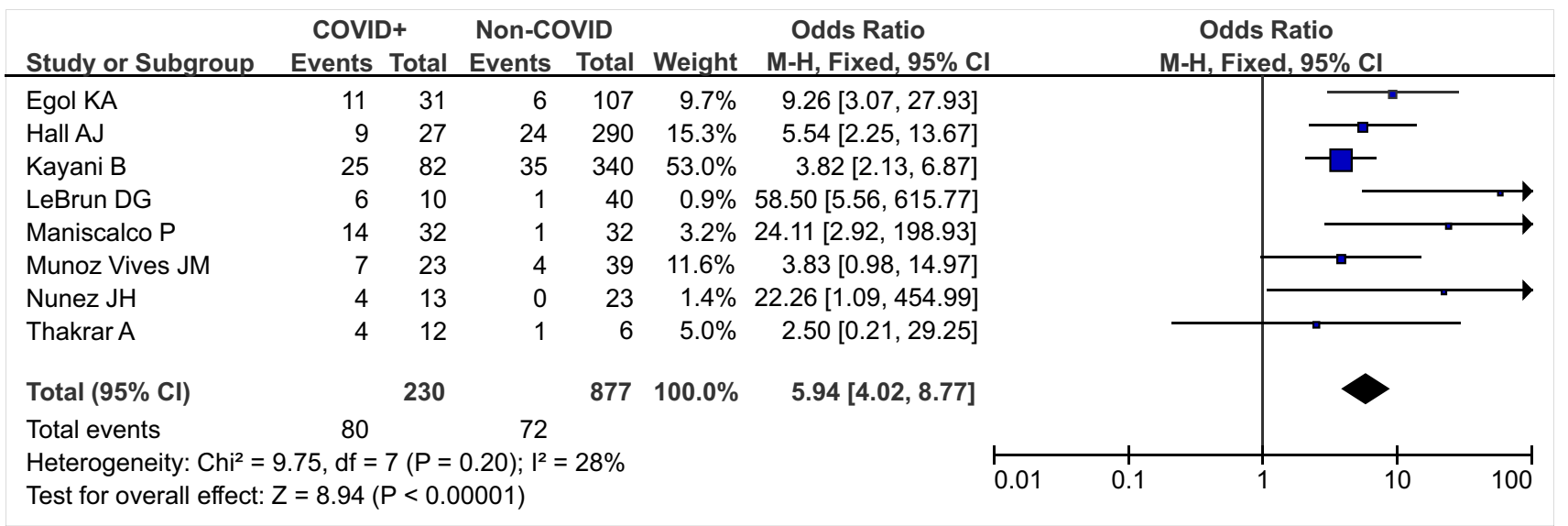

Fig. 4 Sensitivity analysis-Odds ratios for mortality in patients with COVID-19 versus non-COVID (without patients who were not tested) illustrated in a Forest plot. The pooled estimate was generated using a fixed effect model

\section{Meta-analysis}

\section{The effect of COVID-19 on mortality in hip fracture patients} (Fig. 2).

The eight studies included in the analysis all showed an increased mortality among patients infected with COVID19. The range of ORs was large, from 3.82 (95\% CI $2.13-6.87, p=0.0)$ in the study by Kayani B to 72.00 (95\% CI $6.87-754.90, p=0.0004$ ) in the study by Lebrun DG. The analysis had an overall $p$ value less than 0.00001 , and a pooled OR of 6.70 (95\% CI 4.64-9.68).

The sensitivity analyses (Figs. 3, 4) showed slightly altered ORs. The pooled OR was increased to 6.83 (95\% CI 4.70-9.92) in the analysis, where the COVID-19 suspected patients were deducted from the group of confirmed positive patients (Fig. 3). The analysis excluding patients who were not tested (Fig. 4), showed a decreased pooled OR of 5.94 (95\% CI 4.02-8.77).

\section{Discussion}

The meta-analysis points towards an increased mortality among hip fracture patients with perioperative COVID-19 infection. The results are consistent with the findings in the meta-analysis by Lim MA et al., who found an increase in relative risk of 7.45 [15].

It is possible that a change in surgical procedures during the epidemic had an influence on the increased mortality: different safety measures were taken when treating COVID-19-positive patients and in some areas, patients were discharged earlier than they would have been under normal circumstances [5]. In some cases, the epidemic 
delayed the surgical treatment $[6,7,9]$, and changed fixation methods or non-operative management have even been suggested. [16, 17] Especially, the delay of surgery is known to affect the survival outcome of hip fracture patients: a meta-analysis including 2,57,367 hip fracture patients found that 30 days mortality increases by $41 \%$ if surgery is delayed beyond $48 \mathrm{~h}$ [18]. The impact of delay to surgery in hip fracture patients infected with COVID-19 is probably even greater given their additional morbidity, making it important to avoid unnecessary delay to surgery also in these patients.

The sensitivity analysis showed only minor changes in odds ratios. This confirms that our assumption to put patients who were not tested under the group of confirmed negative patients and the COVID-19 suspected patients under the group of confirmed positive patients, did not have any major effect on the meta-analysis. However, it is important to note the possible difficulty in distinguishing the effect of COVID-19 infection from similar symptoms and comorbidities, when suspected and confirmed patients are considered as one group. This can be exemplified by the data collected in the study by Muñoz Vives et al : only patients with respiratory symptoms were tested for COVID-19. The 14 day mortality among the group of patients who had a negative test was 10.3 , and only $2.7 \%$ among the group that were not tested. Some of the patients who had symptoms, but a negative test may be considered "suspected" by some studies (e.g., Egol KA et al.). Due to the marked difference in mortality, there is a risk that part of the measured excess mortality among COVID-19 patients is linked to unspecific respiratory symptoms, when adding the group of suspected patients to the group of confirmed positive patients. This, however, is done due to a lack of tests and to the low sensitivity of the performed tests in the early stages of the epidemic, in which the data were collected [19].

During the outbreak, different diagnostic tests were used to identify COVID-19 infections. In a study from Piacenza [7], Italy, patients with symptoms of COVID-19 (fever, dry cough, tiredness, etc.) were diagnosed by different types of tests: chest CT scan, nasopharyngeal swab, CUS (carotid ultrasound) and LUS (lung ultrasound). The most widely used method to detect coronavirus is real-time reverse-transcription polymerase chain reaction (real-time RT-PCR) [20]. Some of the patients with symptoms in Piacenza were only diagnosed COVID-19-positive through a chest CT scan [7]. A retrospective study from China showed that $56 \%$ confirmed positive patients (real-time RT-PCR) had a normal CT, imaged a few days after symptom onset [21]. "Chest CT therefore has limited sensitivity and negative predictive value early after symptom onset, and is thereby unlikely a reliable standalone tool to rule out COVID19 infection" [21]. This means that some patients in our meta-analysis, like the patients in Piacenza, may have had another infectious disease, due to its COVID-19 confirmation through a CT scan.

Many aspects of the COVID-19 pandemic still remain unclear. This meta-analysis including all the data currently available on the topic shows a markedly increased mortality among hip fracture patients supported by a low $95 \%$ confidence interval (95\% CI 4.64-9.68). This illustrates the importance of further care and attention to this vulnerable group of patients in the future.

\section{Conclusion}

Perioperative infections with COVID-19 in hip fracture patients are correlated with a significantly increased mortality. The meta-analysis showed a pooled odds ratio of 6.70 (95\% CI 4.64-9.68).

Author's contributions JF: conceptualization, investigation, data analysis, drafting and revision of the final manuscript, approved the final manuscript, TJ: conceptualization, investigation, data analysis, drafting and revision of the final manuscript, approved the final manuscript, HLJ: conceptualization, data analysis, approved the final manuscript, JBL: conceptualization, approved the final manuscript.

\section{Declarations}

Conflict of interest No conflicts of interest were declared for this study.

\section{References}

1. Richardson S, Hirsch JS, Narasimhan M, et al. Presenting characteristics, comorbidities, and outcomes among 5700 patients hospitalized With COVID-19 in the New York city area. JAMA. 2020;323(20):2052-9.

2. Benazzo F, Rossi SMP, Maniscalco P, et al. The orthopaedic and traumatology scenario during Covid-19 outbreak in Italy: chronicles of a silent war. Int Orthop. 2020;44(8):1453-9.

3. Lauritzen JB. Hip fractures. Epidemiology, risk factors, falls, energy absorption, hip protectors, and prevention. Dan Med Bull. 1997;44(2):155-68.

4. Johnell O, Kanis JA. An estimate of the worldwide prevalence, mortality and disability associated with hip fracture. Osteoporos Int. 2004;15(11):897-902.

5. LeBrun DG, Konnaris MA, Ghahramani GC, et al. Hip fracture outcomes during the COVID-19 pandemic: early results from New York. J Orthop Trauma. 2020;34(8):403-10.

6. Kayani B, Onochie E, Patil V, et al. The effects of COVID-19 on perioperative morbidity and mortality in patients with hip fractures. Bone Joint J. 2020;102-B(9):1136-45.

7. Maniscalco P, Poggiali E, Quattrini F, et al. Proximal femur fractures in COVID-19 emergency: the experience of two Orthopedics and Traumatology Departments in the first eight weeks of the Italian epidemic. Acta Biomed. 2020;91(2):89-96.

8. Nuñez JH, Sallent A, Lakhani K, et al. Impact of the COVID-19 pandemic on an emergency traumatology service: experience at a Tertiary Trauma Centre in Spain. Injury. 2020;51(7):1414-8. 
9. Egol KA, Konda SR, Bird ML, et al. Increased mortality and major complications in hip fracture care during the COVID19 pandemic: a New York city perspective. J Orthop Trauma. 2020;34(8):395-402.

10. Muñoz Vives JM, Jornet-Gibert M, Cámara-Cabrera J, et al. Mortality rates of patients with proximal femoral fracture in a worldwide pandemic: preliminary results of the Spanish HIP-COVID Observational Study. J Bone Joint Surg Am. 2020;102(13):e69.

11. Thakrar A, Chui K, Kapoor A, Hambidge J. Thirty-day mortality rate of patients with hip fractures during the COVID-19 pandemic: a single centre prospective study in the United Kingdom. J Orthop Trauma. 2020;34(9):e325-9.

12. Hall AJ, Clement ND, Farrow L, et al. IMPACT-Scot report on COVID-19 and hip fractures. Bone Joint J. 2020;102-B(9):1219-28.

13. Moher D, Liberati A, Tetzlaff J, Altman DG. Preferred reporting items for systematic reviews and meta-analyses: the PRISMA statement. PLoS Med. 2009;6(7):e1000097.

14. Higgins JPT, Thomas J, Chandler J, Cumpston M, Li T, Page MJ, Welch VA, editors. Cochrane handbook for systematic reviews of interventions version 6.2. Cochrane; 2021. www.training.cochr ane.org/handbook.

15. Lim MA, Pranata R. Coronavirus disease 2019 (COVID-19) markedly increased mortality in patients with hip fracturea systematic review and meta-analysis. J Clin Orthop Trauma. 2020;12(1):44.

16. Cronin M, Mullins M, Pathmanaban P, Williams P, Dodd M (2020) COVID-19 causes a SHiFT in the sands for proximal femoral fracture management? https://www.boa.ac.uk/polic y-engagement/journal-of-trauma-orthopaedics/journal-of-traum a-orthopaedics-and-coronavirus/covid-19-causes-a-shift-in-thesands-for-proximal.html.

17. Mitkovic MM, Bumbasirevic M, Milenkovic S, Gajdobranski D, Bumbasirevic V, Mitkovic MB. Influence of coronavirus disease 2019 pandemic state of emergency in orthopaedic fracture surgical treatment. Int Orthop. 2020. https://doi.org/10.1007/s0026 4-020-04750-3.

18. Shiga T, Wajima Z, Ohe Y. Is operative delay associated with increased mortality of hip fracture patients? Systematic review, meta-analysis, and meta-regression. Can J Anaesth. 2008;55(3):146-54.

19. Hirotsu Y, Mochizuki H, Omata M. Double-quencher probes improve detection sensitivity toward Severe Acute Respiratory Syndrome Coronavirus 2 (SARS-CoV-2) in a reverse-transcription polymerase chain reaction (RT-PCR) assay. J Virol Methods. 2020;284:113926.

20. Mathuria JP, Yadav R, Rajkumar. Laboratory diagnosis of SARSCoV-2-a review of current methods. J Infect Public Health. 2020;13(7):901-5.

21. Bernheim A, Mei X, Huang M, et al. Chest CT findings in coronavirus disease-19 (COVID-19): relationship to duration of infection. Radiology. 2020;295(3):200463. 